\title{
TRIGONOMETRIC SERIES WITH POSITIVE PARTIAL SUMS
}

\author{
BY Y. KATZNELSON
}

\section{Communicated by W. Rudin, April 12, 1965}

The following problem was proposed by J. E. Littlewood about 15 years ago: Let $S(x)=\sum_{-\infty}^{\infty} c_{n} e^{i n x}$ be a trigonometric series having the property that all its partial sums are positive. Is such a series necessarily a Fourier series? The purpose of this note is to show that such is not the case. It is well known that such a series must be a FourierStieltjes series, and, as was shown by H. Helson, even the weaker condition

$$
\int\left|S_{n}(x)\right| d x<\text { const., } \quad\left(S_{n}(x)=\sum_{-n}^{n} c_{j} e^{i j x}\right)
$$

implies $c_{n}=o(1)$ (cf. Zygmund [2, p. 286]). It has been shown by Mary Weiss [1] that condition (1) does not imply that $S(x)$ is a Fourier series.

LEMma 1. There exists a constant $\alpha>0$ such that for every $\epsilon>0$ there exists a real valued trigonometric polynomial $P_{\epsilon}(x)$, with vanishing constant coefficient, having the properties:

(i) $|\hat{P}(j)|<\epsilon$,

(ii) $P_{\epsilon}(x)>\alpha$ on a set of measure $>\alpha$,

(iii) The absolute values of the partial sums of $P_{\epsilon}(x)$ are less than $1 / 2$.

Proof. There exists a constant $C$ such that $\left|(1 / \sqrt{ } N) \sum_{1}^{N} e^{i n \log n} e^{\text {inx }}\right|$ $<C$ (cf. Zygmund [2, p. 199]). Take $N>\epsilon^{-2}$ and $P_{\epsilon}(x)$ $=\operatorname{Re}\left((1 / 2 C \sqrt{ } N) \sum_{1}^{N} e^{i n \log { }^{n}} e^{i n x}\right)$. Properties (i) and (iii) are obvious. Property (ii) follows from the fact that

$$
\left\|P_{\epsilon}\right\|_{L^{2}}=\frac{1}{2 \sqrt{ }(2) C}, \quad \sup \left|P_{\epsilon}(x)\right| \leqq \frac{1}{2} .
$$

We shall also need the following lemma:

Lemma 2. Let $f_{j}(x)$ be real valued trigonometric polynomials satisfying:

(a) $\hat{f}(0)=0$,

(b) $f_{j}(x)>\epsilon$ on a set of measure $>\alpha$,

(c) $\left|f_{j}(x)\right|<1 / 2$.

Then, if $\lambda_{j} \rightarrow \infty$ fast enough, the product 


$$
\prod_{1}^{\infty}\left(1-f_{j}\left(\lambda_{j} x\right)\right)
$$

converges weakly to a singular measure.

Proof. Our first condition on the growth of $\lambda_{n}$ is:

$$
\lambda_{n}>3 \text { times the degree of } \prod_{1}^{n-1}\left(1-f_{j}\left(\lambda_{j} x\right)\right)
$$

which implies that the constant term of $\prod_{1}^{n}\left(1-f_{j}\left(\lambda_{j} x\right)\right)$ is 1 for all $n$. Since the partial products are positive, this implies that the (formal) product (2) is a Fourier-Stieltjes series of a positive measure $\mu$. All that we have to do now is follow the lines of the proof of Theorem V.7.6, p. 209 in Zygmund [2]: We notice first that the partial products $\prod_{1}^{n}\left(1-f_{j}\left(\lambda_{j} x\right)\right)$ are partial sums of $S(d \mu)$ followed by long gaps. As is well known, this implies $\prod_{1}^{n}\left(1-f_{j}\left(\lambda_{j} x\right)\right) \rightarrow \phi(x)$ a.e. where $\phi(x) d x$ is the absolutely continuous part of $\mu$; but if $\lambda_{n}$ grows fast enough (b) implies that the only limit $\prod_{1}^{n}\left(1-f_{j}\left(\lambda_{j} x\right)\right)$ can converge to a.e. is zero.

The Example. We take $S(x)=\prod_{1}^{\infty}\left(1-P_{\epsilon_{j}}\left(\lambda_{j} x\right)\right)$.

The $P_{e_{j}}$ are the polynomials defined in Lemma 1 , with

$$
0<\epsilon_{j}<2^{-j-2}\left\|\prod_{1}^{j-1}\left(1-P_{\epsilon_{k}}\left(\lambda_{k} x\right)\right)\right\|_{A}^{-1}
$$

(where $\|g\|_{A}=\sum|\hat{g}(n)|$ ) and $\lambda_{j} \rightarrow \infty$ rapidly enough so that

(a) $\lambda_{j}>3$ times the degree of $\prod_{1}^{j-1}\left(1-P_{\epsilon_{k}}\left(\lambda_{k} x\right)\right)$ and

(b) $S(x)$ is the Fourier-Stieltjes series of a singular measure (Lemma 2).

From (a) above it follows that a partial sum of $S(x)$ has necessarily the form $\prod_{1}^{q}\left(1-P_{\epsilon_{j}}\left(\lambda_{j} x\right)\right)$ times a partial sum of $\left(1-P_{e_{q+1}}\left(\lambda_{q+1} x\right)\right)$ plus two groups of terms each having the form

$$
P_{\epsilon q+1}(k) e^{i k x} \text { times some terms from } \prod_{1}^{q}\left(1-P_{e_{j}}\left(\lambda_{j} x\right)\right) .
$$

By (iii) $\prod_{1}^{q}\left(1-P_{\epsilon_{j}}\left(\lambda_{j} x\right)\right)>2^{-q}$ and the partial sums of $\left(1-P_{e_{q+1}}\left(\lambda_{q+1} x\right)\right)$ are $>1 / 2$ and by (4) the sum of the remaining terms is bounded by $2^{-\alpha-2}$, hence the partial sums of $S(x)$ are positive.

\section{REFERENCES}

1. M. Weiss, On a problem of Littlewood, J. London Math. Soc. 34 (1959), 217-221.

2. A. Zygmund, Trigonometric series, Vol. 1, University Press, Cambridge, 1959.

STANFORD UNIVERSITY 